\title{
The Role of Clinical Trials in Preclinical Alzheimer's Disease Drug Development Programs
}

\section{J. Cummings}

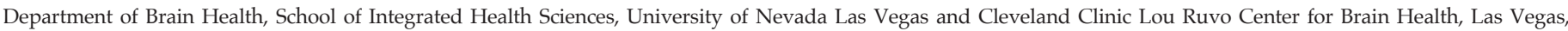
Nevada, USA

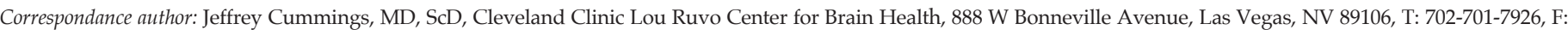
702-722-6584, E: jcummings@cnsinnovations.com

\section{Introduction}

A lzheimer's disease (AD) has a 15-20 year preclinical phase during which the individuals have normal cognition by conventional measures and have state measures of AD pathology including elevated levels of brain amyloid when assessed with positron emission tomography (PET) and abnormally decreased levels cerebrospinal fluid (CSF) amyloid beta-protein (AB) and increased levels of total tau and hyperphosphorylated tau (p-tau) (1, 2 ). Recognition of this long-preclinical phase and the presence of biomarker changes affords, the opportunity to plan secondary prevention trials to prevent or delay the onset of cognitive impairment and progression to dementia. The US Food and Drug Administration (FDA) facilitated planning of clinical trials for participants in the preclinical phase of AD by defining two stages of the preclinical period: in stage 1 participants have characteristic pathophysiologic changes of AD but no evidence of clinical impact; in stage 2 participants have characteristic pathophysiologic changes of $\mathrm{AD}$ and subtle detectable abnormalities on sensitive neuropsychological measures, but no functional impairment. Stage 3 participants are no longer in the preclinical phase of $\mathrm{AD}$; they have mild cognitive impairment (MCI)/prodromal $\mathrm{AD}$ with characteristic pathophysiologic changes of $\mathrm{AD}$, subtle or more apparent detectable abnormalities on sensitive neuropsychological measures, and mild but detectable functional impairment. Stages 4, 5, and 6 describe patients with mild, moderate and severe $\mathrm{AD}$ dementia. Biomarker, clinical definitions, and regulatory engagement have set the stage for planning and conduct of trials in preclinical AD.

\section{Drug Development}

Drug development programs are constructed around current understanding of target biology, treatment pharmacology, and clinical trial design with the intent of reducing the risk of a negative outcome and optimizing the opportunity to observe a drug-placebo difference with the intervention. Principles of good clinical practice for drug development indicate that identifying a wellsupported target, discovering an agent with acceptable pharmacokinetic properties, demonstrating target engagement in Phase 2, implementing a well conducted trial in an appropriate population using qualified clinical and biomarker outcomes in Phase 3, and demonstrating safety throughout the program create the circumstances most likely to succeed in advancing an agent toward approval (3).

These conditions are difficult to meet in preclinical AD than in other disease stages making it more challenging to successfully advance a drug candidate.

\section{Population}

The efficacy of disease-modifying therapies (DMTs) is demonstrated by showing a drug-placebo difference on biomarkers or clinical measures. Decline or worsening of the placebo group is critical to this comparison. Most people in the preclinical phase of AD with brain amyloidosis will not progress to AD dementia in their lifetime and will exhibit no or limited cognitive decline. An 80 year old person with a positive amyloid PET and eligible for a clinical trial has a $19.1 \%$ risk of developing AD dementia in their lifetime. A 65 year old with a positive amyloid PET has a $29.3 \%$ risk for progressing to AD dementia in their lifetime (4). These examples demonstrate that progression to AD dementia in the remaining lifetime of potential trial participants occurs in a minority of individuals and progression sufficient to demonstrate a drug-placebo difference in the course of a clinical trial is unlikely.

Sensitive measures of cognitive decline can demonstrate change in cognition in preclinical populations corresponding to the FDA stage 2. Using the Preclinical Alzheimer's Cognitive Composite (PACC), a preclinical trial tool, there is a 1.51 point decline over four years. The mean change in the Mini Mental State over 4 years is 0.56 points and the Clinical Dementia Rating - Sum of boxes declines 0.23 points (5). Overall, at 
4 years, $14.5 \%$ of amyloid positive individuals progressed from cognitively normal to MCI. Similarly, in a cohort followed for 30 months there was no difference in decline of those with and without brain amyloid on the Clinical Dementia Rating or the Mini Mental Status Examination. Four of 88 had progressed from cognitively normal to prodromal AD (6).

These small changes over observation periods comparable to the duration of trials of preclinical populations indicate that very large sample sizes observed for extended periods of time would be needed to demonstrate a drug-placebo difference.

When cognitively normal individuals are asked about preferred outcomes for treatment to avoid cognitive decline, they identify maintaining everyday functioning, enjoying life, keeping a sense of identity and maintaining relationship and social connections as priorities (7). Current assessment approaches in preclinical trials do not measure these preferred outcomes.

\section{Biomarkers}

The FDA Guidance indicates than an accelerated approval might be possible for a treatment for preclinical individuals based on biomarker outcomes (8). The guidance states that in Stage 1 preclinical disease, an effect on the characteristic biomarker changes of $\mathrm{AD}$ may serve as the basis for an accelerated approval --- with a post-approval requirement for a study to confirm the predicted clinical benefit --- if the biomarker effects are reasonably likely to predict clinical benefit. The FDA notes that at present no biomarker changes have been shown to be reasonably likely to predict clinical benefit.

For Stage 2 individuals, a pattern of putatively beneficial effects across multiple individual neuropsychological tests or a large magnitude of effect on sensitive measures of neuropsychological performance may increase their persuasiveness. It is expected that neuropsychological benefits would be supported by similarly persuasive effects on biomarkers (8). Given the small changes observed on sensitive neuropsychological measures in preclinical populations, demonstration of "large magnitude" effects is daunting.

Tau PET may serve as an important biomarker for preclinical trials. The presence of an elevated tau burden on PET is associated with more rapid cognitive decline (9). Power calculations based on tau PET measures demonstrate that to detect a $25 \%$ annual slowing of disease progression on this measure with $80 \%$ power (two-sided alpha of 0.05), using an early AD meta-region of interest analysis with partial volume correction, would require 1228 patients. A whole brain meta-region of interest analysis would require 3512 participants (10). With tau PET substantial sample sizes are required to show drug-placebo differences in clinical trials with preclinical participants.

Biomarkers characterize the biology of AD (11). Their longitudinal change in preclinical populations is limited, and they do not de-risk drug development in this early phase of the AD continuum.

\section{Treating Early}

Preventing cognitive impairment and the associated functional compromise, caregiver distress, costs, and accelerated mortality are critical goals for those at risk for $\mathrm{AD}$ and for clinicians and drug developers striving to maintain individuals at the highest level of function for the longest period of time. Treatment responsiveness may be improved by treating $\mathrm{AD}$ in the preclinical phase of the disease when pathological abnormalities are more limited and have been present for a shorter period of time (12). Cognitive resilience, however, may persist late into AD. Current therapies are approved for patients with moderate to severe AD demonstrating the brain's ability to respond in advanced states. This suggests that treating early may not be required for successful drug development.

Responsiveness to treatment with anti-amyloid therapies may not be limited to the earliest phases of AD. Aducanumab produced equivalent reductions in brain amyloid in mild AD dementia (global Clinical Dementia Rating of 1.0) and in prodromal AD (global Clinical Dementia Rating of 0.5), and clinical benefit correlated with amyloid removal $(13,14)$. This suggests that the failure of drug candidates in later phase disease may have been due to lack of efficacy of the agents; use of more effective agents later in the course of the disease is plausible.

\section{Post-Approval Use}

The cost and effort of developing drugs is justified if the ensuing treatments are welcomed and used by those for whom the treatments were developed. In a survey of cognitively normal individuals to determine if they would participate in a trial of an approved medication intended to lower the risk of dementia, $32.4 \%$ said they were very likely to participate, $51.4 \%$ were neutral, and $16.2 \%$ said it was very unlikely (15).

Payers will be required to pay for medications for cognitively normal individuals at risk for dementia to create a market for agents to treat individuals in the preclinical phases of AD. Payers preferentially use number-needed-to-treat (NNT) and number-needed-toharm (NNH) approaches to determine the cost-utility of medications proposed for the insured population. Given the high rate of non-progression in amyloid PET positive individuals and the high cost of more precise characterizations (e.g, amyloid PET plus tau PET) as well as the risk associated with some infusion approaches (16), the NNT and NNH calculations are likely to be unfavorable, and payers may question the value of the investment. The availability of blood tests to identify 
preclinical persons at high risk for progression and the emergence of safer medications will impact the NNT/ $\mathrm{NNH}$ discussion $(17,18)$.

Complicating the post-approval use of treatments for preclinical at-risk individuals is the lack of sufficient diagnostic and therapeutic capacity. Recent surveys of the ability to respond to the approval of new treatments for prodromal $\mathrm{AD}$ show that there are too few clinicians skilled in diagnosis, too few PET facilities to establish the amyloid status, and too few infusion centers to treat all treatment candidates if the therapy is administered intravenously $(19,20)$. These same considerations apply to persons with preclinical disease; the larger numbers of those in the preclinical phase of $\mathrm{AD}$ would make the deficiencies relatively more severe.

\section{Conclusion}

Clinical trials in preclinical disease are important experiments to determine if $\mathrm{AD}$-related cognitive decline can be prevented to deferred. These trials will provide critical information regarding the clinical and biomarker features of the earliest phases of the AD continuum and their response to intervention. The lack of progression to dementia of most people in the preclinical phase, the limited cognitive decline observed in the preclinical period, the absence of surrogate biomarkers on which an accelerated approval could be based, the limited interest of individuals in the preclinical phase to pursue treatment, and the challenges to obtaining payment combine to make development programs focusing on preclinical populations high risk propositions.

Disclosures: JC has provided consultation to Acadia, Actinogen, AgeneBio, Alkahest, Alzheon, Annovis, Avanir, Axsome, Biogen, BioXcel, Cassava, Cerecin, Cerevel, Cortexyme, EIP Pharma, Eisai, Foresight, GemVax, Genentech, Green Valley, Grifols, Karuna, Merck, Novo Nordisk, Otsuka, Resverlogix, Roche, Samumed, Samus, Signant, Suven, Third Rock, and United Neuroscience pharmaceutical and assessment companies. Dr. Cummings has stock options in ADAMAS, AnnovisBio, MedAvante, BiOasis. Dr Cummings is supported by Keep Memory Alive (KMA); NIGMS grant P20GM109025; NINDS grant U01NS093334; and NIA grant R01AG053798.

Open Access: This article is distributed under the terms of the Creative Commons Attribution 4.0 International License (http:/ / creativecommons.org/ licenses/by/4.0/), which permits use, duplication, adaptation, distribution and reproduction in any medium or format, as long as you give appropriate credit to the original author(s) and the source, provide a link to the Creative Commons license and indicate if changes were made.

\section{References}

1. Villemagne VL, Burnham S, Bourgeat P, Brown B, Ellis KA, Salvado O, et al. Amyloid beta deposition, neurodegeneration, and cognitive decline in sporadic Alzheimer's disease: a prospective cohort study. Lancet Neurol. 2013;12:357-367.

2. Fagan AM, Xiong C, Jasielec MS, Bateman RJ, Goate AM, Benzinger TL, et al Longitudinal change in CSF biomarkers in autosomal-dominant Alzheimer's disease. Sci Transl Med. 2014;6:226ra230.

3. Cummings J, Feldman HH, Scheltens P. The «rights» of precision drug development for Alzheimer's disease. Alzheimers Res Ther. 2019;11:76.

4. Brookmeyer R, Abdalla N. Estimation of lifetime risks of Alzheimer's disease dementia using biomarkers for preclinical disease. Alzheimers Dement. 2018;14:981-988

5. Donohue MC, Sperling RA, Petersen R, Sun CK, Weiner MW, Aisen PS, et al. Association between elevated brain amyloid and subsequent cognitive decline among cognitively normal persons. JAMA. 2017;317:2305-2316.

6. Dubois B, Epelbaum S, Nyasse F, Bakardjian H, Gagliardi G, Uspenskaya O, et al. Cognitive and neuroimaging features and brain beta-amyloidosis in individuals at risk of Alzheimer's disease (INSIGHT-preAD): a longitudinal observational study. Lancet Neurol. 2018;17:335-346.

7. Watson J, Saunders S, Muniz Terrera G, Ritchie C, Evans A, Luz S, et al. What matters to people with memory problems, healthy volunteers and health and social care professionals in the context of developing treatment to prevent Alzheimer's dementia? A qualitative study. Health Expect. 2019;22:504-517.

8. Food and Drug Administration. Early Alzheimer's disease: developing drugs for treatment guidance for industry. U.S. Department of Health and Human Services Food and Drug Administration Center for Drug Evaluation and Research (CDER) Center for Biologics Evaluation and Research (CBER). February, 2018.

9. Hanseeuw BJ, Betensky RA, Jacobs HIL, Schultz AP, Sepulcre J, Becker JA, et al. Association of amyloid and tau with cognition in preclinical Alzheimer disease: a longitudinal study. JAMA Neurol. 2019.

10. Jack CR, Jr., Wiste HJ, Schwarz CG, Lowe VJ, Senjem ML, Vemuri P, et al. Longitudinal tau PET in ageing and Alzheimer's disease. Brain. 2018;141:15171528.

11. Jack CR, Jr., Bennett DA, Blennow K, Carrillo MC, Dunn B, Haeberlein SB, et al. NIA-AA Research Framework: toward a biological definition of Alzheimer's disease. Alzheimers Dement. 2018;14:535-562.

12. Mehta D, Jackson R, Paul G, Shi J, Sabbagh M. Why do trials for Alzheimer's disease drugs keep failing? A discontinued drug perspective for 2010-2015. Expert Opin Investig Drugs. 2017;26:735-739.

13. Sevigny J, Chiao P, Bussiere T, Weinreb PH, Williams L, Maier M, et al. The antibody aducanumab reduces Abeta plaques in Alzheimer's disease. Nature. 2016;537:50-56.

14. Sevigny J, Chiao P, Bussiere T, Weinreb PH, Williams L, Maier M, et al. Addendum: The antibody aducanumab reduces Abeta plaques in Alzheimer's disease. Nature. 2017;546:564.

15. Nuno MM, Gillen DL, Dosanjh KK, Brook J, Elashoff D, Ringman JM, et al Attitudes toward clinical trials across the Alzheimer's disease spectrum. Alzheimers Res Ther. 2017;9:81.

16. Sperling RA, Jack CR, Jr., Black SE, Frosch MP, Greenberg SM, Hyman BT, et al. Amyloid-related imaging abnormalities in amyloid-modifying therapeutic trials: recommendations from the Alzheimer's Association Research Roundtable Workgroup. Alzheimers Dement. 2011;7:367-385.

17. Nakamura A, Kaneko N, Villemagne VL, Kato T, Doecke J, Dore V, et al. High performance plasma amyloid-beta biomarkers for Alzheimer's disease. Nature. 2018;554:249-254.

18. Thijssen EH, La Joie R, Wolf A, Strom A, Wang P, Iaccarino L, et al. Diagnostic value of plasma phosphorylated tau181 in Alzheimer's disease and frontotemporal lobar degeneration. Nat Med. 2020;26:387-397.

19. Liu JL, Hlavka JP, Hillestad R, Mattke S. Assessing the preparedness of the U.S. health care system infrastructure for an Alzheimer's treatment. RAND Corporation. Los Angeles, CA2017.

20. Ritchie CW, Russ TC, Banerjee S, Barber B, Boaden A, Fox NC, et al. The Edinburgh Consensus: preparing for the advent of disease-modifying therapies for Alzheimer's disease. Alzheimers Res Ther. 2017;9:85. 